\title{
Urine Albumin
}

National Cancer Institute

\section{Source}

National Cancer Institute. Urine Albumin. NCI Thesaurus. Code C121532.

Albumin protein in urine samples, which is a marker for kidney dysfunction. Persistently elevated concentrations of albumin in the urine can be diagnostic for kidney damage caused by diabetes, high blood pressure, heart failure, cirrhosis, or lupus. 